Submitted: 2007 July 21; Accepted: 2007 August 10

\title{
Constraining the Type Ia Supernova Progenitor: The Search for Hydrogen in Nebular Spectra ${ }^{1}$
}

\author{
Douglas C. Leonard \\ Department of Astronomy, San Diego State University, San Diego, CA 92182-1221 \\ leonard@sciences.sdsu.edu
}

\begin{abstract}
Despite intense scrutiny, the progenitor system(s) that gives rise to Type Ia supernovae remains unknown. The favored theory invokes a carbon-oxygen white dwarf accreting hydrogen-rich material from a close companion until a thermonuclear runaway ensues that incinerates the white dwarf. However, simulations resulting from this single-degenerate, binary channel demand the presence of low-velocity $\mathrm{H} \alpha$ emission in spectra taken during the late nebular phase, since a portion of the companion's envelope becomes entrained in the ejecta. This hydrogen has never been detected, but has only rarely been sought. Here we present results from a campaign to obtain deep, nebular-phase spectroscopy of nearby Type Ia supernovae, and include multi-epoch observations of two events: SN 2005am (slightly subluminous) and SN 2005cf (normally bright). No $\mathrm{H} \alpha$ emission is detected in the spectra of either object. An upper limit of $0.01 M_{\odot}$ of solar abundance material in the ejecta is established from the models of Mattila et al. (2005) which, when coupled with the mass-stripping simulations of Marietta et al. (2000) and Meng et al. (2007), effectively rules out progenitor systems for these supernovae with secondaries close enough to the white dwarf to be experiencing Roche lobe overflow at the time of explosion. Alternative explanations for the absence of $\mathrm{H} \alpha$ emission, along with suggestions for future investigations necessary to confidently exclude them as possibilities, are critically evaluated.
\end{abstract}

Subject headings: binaries: symbiotic — circumstellar matter - supernovae: general - supernovae: individual (SN 2005am, SN 2005cf) — white dwarfs

\footnotetext{
${ }^{1}$ Some of the data presented herein were obtained at the W.M. Keck Observatory, which is operated as a scientific partnership among the California Institute of Technology, the University of California and
} 


\section{Introduction}

Ever since the currently favored single-degenerate, binary channel was proposed as the progenitor system for Type Ia supernovae (Whelan \& Iben 1973), all models of the impact of the exploded white dwarf (WD) on the secondary star have indicated that significant amounts of solar-abundance material, stripped from the secondary's envelope, become entrained in the ejecta (Wheeler et al. 1975; Frvxell \& Arnett 1981; Taam \& Frvxell 1984; Chugai 1986; Livne et al. 1992; Marietta et al. 2000; Meng et al. 2007). Observational evidence for this material, however, still eludes us (Mattila et al. 2005), and serves as one reminder among many that we still lack direct observational proof for the single-degenerate scenario (Branch et al. 1995; Livio 2001).

The most detailed theoretical investigation of the expected amount and distribution of stripped material within a young Type Ia supernova (SN Ia) remnant is that of Marietta et al. (2000), who studied the problem with two-dimensional numerical simulations. Four basic progenitor systems were investigated, including three with secondaries (a main-sequence, subgiant, or red giant star) close enough for mass-transfer to occur through Roche lobe overflow (RLOF), and one containing a secondary (a red giant) donating material through a strong stellar wind - the symbiotic case. All secondaries were given masses of $\sim 1 M_{\odot}$ at the time of the explosion and were placed either just within (the RLOF cases), or just beyond (the symbiotic case), the limiting distance from the WD within which RLOF can occur (i.e., $a / R=3$, where $a$ is the orbital separation in units of the secondary star's radius, $R$; see Eggleton 1983). Three additional systems, in which a main-sequence secondary was placed too far away to experience RLOF (thus rendering it an unlikely progenitor system) were also included to establish the scaling between orbital separation and amount of stripped material.

The numerical results of the Marietta et al. (2000) study confirmed predictions from earlier analytic work (e.g., Wheeler et al. 1975; Chugai 1986) that substantial material is indeed stripped, with the amount ranging from a minimum of $0.15 M_{\odot}$ for a close $(a / R=3)$ main-sequence secondary, up to nearly the entire envelope $\left(\sim 0.5 M_{\odot}\right)$ for a similarly placed red giant. Increasing the orbital separation beyond the RLOF limit in the Marietta et al. (2000) simulations resulted in a dramatic decrease in the amount of stripped material: For $a / R=12$, a main-sequence secondary loses only $0.0018 M_{\odot}$. However, since such

the National Aeronautics and Space Administration. The Observatory was made possible by the generous financial support of the W.M. Keck Foundation. Additional observations were obtained at the Gemini Observatory, which is operated by the Association of Universities for Research in Astronomy, Inc., under a cooperative agreement with the NSF on behalf of the Gemini partnership: the National Science Foundation (United States), the Particle Physics and Astronomy Research Council (United Kingdom), the National Research Council (Canada), CONICYT (Chile), the Australian Research Council (Australia), CNPq (Brazil) and CONICET (Argentina). 
systems lack an efficient mechanism for mass transfer, they are not considered to be viable SN Ia progenitors. A red giant secondary placed at a similarly distant location, however, could potentially donate mass through a strong stellar wind, making the symbiotic case a possibility if large orbital separations are required (e.g., Munari \& Renzini 1992).

As discussed by Meng et al. (2007), one shortcoming of the Marietta et al. (2000) study is their use of standard solar-model stars for the companion, rather than companions whose structures have been appropriately modified due to having evolved in a binary system (e.g., Eggleton 1973). To investigate the effect this simplification might have had on the results, Meng et al. (2007) use an analytic model to estimate the amount of mass expected to be stripped from a variety of evolved secondaries. (Their analytic approach was first tested using the unevolved secondaries used by Marietta et al. 2000, and was demonstrated to approximate the results obtained numerically.) The result is that the quantity of material expected to be stripped from evolved secondaries is considerably lower than that predicted for standard solar-model companions. In fact, Meng et al. (2007) find that the minimum value for systems experiencing RLOF is diminished from $0.15 M_{\odot}$ to only $0.035 M_{\odot}$. The reduction arises primarily from the pre-explosion mass-loss producing a more compact companion star whose material is more difficult to strip than it is in the unevolved case. Meng et al. (2007) stress, however, that their new values are really lower bounds on the amount of stripped material, since their analytic approach does not consider the thermal energy imparted by the ejecta to the companion envelope, which likely serves to heat and vaporize a portion of it and thereby increase the amount of stripped material (e.g., Fryxell \& Arnett 1981; Mattila et al. 2005). Thus, $0.035 M_{\odot}$ serves as a conservative lower bound on the expected amount of stripped material resulting from their models.

The typical velocity of the stripped material is found in all studies to be far slower than the $\sim 10,000 \mathrm{~km} \mathrm{~s}^{-1}$ velocity that characterizes the bulk of the iron-rich ejecta (Chugai 1986; Marietta et al. 2000; Meng et al. 2007). This has the effect of placing it almost entirely in the central region of the supernova remnant, with the majority of it predicted to be moving with a velocity of under $1,000 \mathrm{~km} \mathrm{~s}^{-1}$ (Marietta et al. 2000). The stripped material is largely confined to the downstream region behind the companion star, where it contaminates a solid angle that ranges from $66^{\circ}$ for the main-sequence companion to $115^{\circ}$ for the red-giant companion (Marietta et al. 2000).

The low velocity of the stripped material renders it undetectable when the fastermoving, iron-rich ejecta are optically thick. However, detailed radiative transfer calculations performed by Mattila et al. (2005) predict that it should become visible at late times, when the outer ejecta have thinned out and become transparent enough to reveal the slower-moving gas in the central regions. The most prominent expected spectral signature of the companion star's stripped material is narrow $\mathrm{H} \alpha$ emission in nebular spectra (Mattila et al. 2005), taken more than $\sim 250$ days after maximum light. The H $\alpha$ emission should be present within $\pm 1,000 \mathrm{~km} \mathrm{~s}^{-1}$ of $\lambda_{0}=6563 \AA$ but, due to the expected 
asymmetry in the distribution of the solar-abundance material, could present an $\mathrm{H} \alpha$ profile ranging from a very narrow spike to a broader emission line in the observed spectrum. Obtaining spectra with high enough resolution to resolve fairly narrow lines is thus a useful component of a targeted search for this $\mathrm{H} \alpha$.

To date, only a few nebular SN Ia spectra have been obtained and $\mathrm{H} \alpha$ has never been detected, although the majority of the spectra lack the spectral resolution and signal-tonoise ratio to place interesting constraints on the companion. The best limits, by far, come from the recent study by Mattila et al. (2005), which constrains hydrogen-rich material in SN 2001el to be $\lesssim 0.03 M_{\odot}$ from a low-resolution $\left(\sim 700 \mathrm{~km} \mathrm{~s}^{-1}\right)$ spectrum obtained 398 days past maximum light.

In an effort to expand and improve on earlier work, both in terms of the number of objects studied as well as the resolution, sensitivity, and temporal coverage of the spectra, we have initiated a program to obtain deep, moderate resolution $\left(\lesssim 150 \mathrm{~km} \mathrm{~s}^{-1}\right.$, or $\sim$ $3 \AA$, at $\mathrm{H} \alpha$ ), late-time spectra of SNe Ia at multiple epochs using the Keck and Gemini telescopes. The first phase of this project has garnered data on two objects: SN 2005am, a slightly subluminous (Li et al. 2006) event and SN 2005cf, an SN Ia of normal brightness (Pastorello et al. 2007). We present and analyze our observations in $\S 2$, discuss the results in $\S$, and conclude in $\S$,

\section{Observations and Analysis}

We obtained a total of five deep nebular-phase spectra: Two for SN 2005am and three for SN 2005cf. Details of the observations are given in Table 1, Following initial processing

of the frames, 2 we extracted all one-dimensional sky-subtracted spectra optimally (Horne 1986) in the usual manner using the apall task within IRAF 3 Each spectrum was then wavelength and flux calibrated, and corrected for continuum atmospheric extinction and telluric absorption bands (Matheson et al. 2000, and references therein). Careful examination of the two-dimensional spectra reveals only faint night-sky emission at the nominal location of $\mathrm{H} \alpha$, and so we deem the potential for contamination at these wavelengths due to improper background ("sky") removal to be negligible.

The final spectra are displayed in Figure 1. To search for $\mathrm{H} \alpha$ emission, or to place limits on the amount of solar-abundance material present in the inner ejecta, we subjected

\footnotetext{
${ }^{2}$ For Gemini observations, the frames were processed using the tasks gprepare, gsreduce, gsflat, and gmosaic in the Gemini IRAF package.

${ }^{3}$ IRAF is distributed by the National Optical Astronomy Observatories, which are operated by the Association of Universities for Research in Astronomy, Inc., under cooperative agreement with the National Science Foundation.
} 
each spectrum to the following analysis procedure.

First, we attempted to place each spectrum on an absolute flux scale. Adjustments to the original flux levels are necessary since most observations were made with a rather narrow slit width compared with the seeing (see Table 1), which makes the flux level susceptible to the effects of seeing variations between the SN observations and those of the flux standard star; some observations were also not obtained under photometric conditions. To produce an approximate absolute flux calibration, then, we computed synthetic photometry on our spectra and compared it with estimates of the $V$ - or $R$-band (depending on the spectral range of the spectrum) magnitudes of the SNe at the spectral epochs. Since none of our spectra cover an entire passband, we extended each spectrum's range by joining it with SN Ia spectra of similar age from the SUSPECT 4 database 5 scaled to match the flux of our spectra in regions of overlap. We then compared our synthetic photometry with estimates of the actual brightnesses of the SNe. Since no published late-time (i.e., beyond $t>100$ days) photometry exists for either SN 2005am or SN 2005cf, but high-quality early-time photometry does (Li et al. 2006; Pastorello et al. 2007), we derived approximate $V$ and $R$ magnitudes of the SNe at our spectral epochs by first extrapolating the early-time light curves to day 200 through comparison with the light curves of SN 2003du (Stanishev et al. 2007), and then applying the late-time Type Ia decay-rates reported by Lair et al. (2006; $\Delta V=1.46 \mathrm{mag} 100$ day $^{-1}, \Delta R=1.54 \mathrm{mag} 100$ day $^{-1}$ ). We then adjusted each of our spectra by multiplying it by the scale factor needed to bring its synthetic magnitude to the estimated actual apparent magnitude. The only spectrum for which we did not apply this procedure was the day 298 epoch of SN 2005am, since the night was photometric, the flux of the SN from one exposure to the next was very consistent (indicating non-varying seeing throughout the observation sequence), and the spectrum of a nearby star (HD 79289) taken immediately after the SN observations was found to have a synthetic $V$ magnitude within $16 \%$ of the value reported by SIMBAD 6 the scale factor for this epoch was thus taken to be the value needed to place HD 79289 on a correct absolute scale. The scale factors applied to all spectra are given in Table 2, Given the uncertainties inherent in our technique, the absolute flux calibrations are probably accurate to $\sim 25 \%$, in general.

We next removed the redshift and rebinned each rest-frame spectrum to $3 \AA \mathrm{bin}^{-1}$, the approximate spectral resolution (see Table 1). For SN 2005am in NGC 2811, we adopted the NASA/IPAC Extragalactic Database (NED) recession velocity of $2,368 \mathrm{~km} \mathrm{~s}^{-1}$, and for SN 2005cf in MCG-01-39-3 we adopted the NED recession velocity of 1, $937 \mathrm{~km} \mathrm{~s}^{-1}$.

\footnotetext{
${ }^{4}$ see http://bruford.nhn.ou.edu/ suspect/index1.html

${ }^{5}$ The specific spectra used for this purpose include SN 1998bu (day 329; Cappellaro et al. 2001), SN 2003cg (day 385; Elias-Rosa et al. 2006), and SN 2002bo (day 375; Benetti et al. 2004).

${ }^{6}$ see http://simbad.u-strasbg.fr/simbad/
} 
We then searched for narrow $\mathrm{H} \alpha$ emission within $\pm 1000 \mathrm{~km} \mathrm{~s}^{-1}( \pm 22 \AA)$ of $\lambda_{0}=6563 \AA$ in each rescaled, rest-frame spectrum by smoothing the spectrum with a second-order Savitsky-Golay smoothing polynomial (Press et al. 1992) of width $\sim 100 \AA$, differencing the smoothed and unsmoothed spectra, and then examining the residuals for narrow emission near $\mathrm{H} \alpha$. This procedure yielded no evidence for narrow $\mathrm{H} \alpha$ emission in any spectrum. Indeed, no unexpected narrow features at any wavelength (including spectral regions near [O I] $\lambda \lambda 6300,6364$ and [Ca II] $\lambda \lambda 7291,7324$, which might be expected to produce features similar to $\mathrm{H} \alpha$ at these epochs from stripped solar-abundance material) were found.

To determine the greatest strength (i.e., equivalent width) of a feature that could have remained undetected at the location of $\mathrm{H} \alpha$ in each spectrum, we adopted the procedure of Leonard \& Filippenko (2001), who report the $3 \sigma$ lower bound on the equivalent width of an undetected feature in a spectrum to be:

$$
W_{\lambda}(3 \sigma)=3 \Delta \lambda \Delta I \sqrt{W_{\text {line }} / \Delta \lambda} \sqrt{1 / B}
$$

where $\Delta \lambda$ is the width of a resolution element (in $\AA$ ), $\Delta I$ is the $1 \sigma$ root-mean-square fluctuation of the flux around a normalized continuum level, $W_{\text {line }}$ is the full-width at halfmaximum of the expected line feature (in $\AA$ ) and $B$ is the number of bins per resolution element in the spectrum. The values for $\Delta \lambda, \Delta I$, and $B$, along with the computed values of $W_{\lambda}(3 \sigma)$, are reported in Table 2. The width of the line feature, $W_{\text {line }}$, was assumed to be $22 \AA$.

While many studies have predicted the existence of narrow $\mathrm{H} \alpha$ in nebular spectra of SNe Ia, only Mattila et al. (2005) gives a quantitative estimate of its expected strength. Thus, to translate our detection thresholds into estimates of the maximum amount of solar-abundance material that could have given rise to an $\mathrm{H} \alpha$ line too weak to have been detected, we rely on this study alone.

In the Mattila et al. (2005) study, the code described by Kozma et al. (2005) is modified to artificially include varying amounts of solar abundance material in the inner $( \pm 1,000 \mathrm{~km}$ $\left.\mathrm{s}^{-1}\right)$ region of the ejecta. The resulting spectra were computed by Mattila et al. (2005) in a time-dependent, one-dimensional, spherically symmetrid 7 manner for an SN Ia 380 days after maximum light. From the parameters given by Mattila et al. (see their Figure 6 and associated discussion), we estimate that $0.05 M_{\odot}$ of solar-abundance material produces a luminosity in the peak of the $\mathrm{H} \alpha$ line of $\sim 3.36 \times 10^{35} \mathrm{erg} \mathrm{s}^{-1} \AA^{-1}$.

Translating predicted $\mathrm{H} \alpha$ luminosity into observed $\mathrm{H} \alpha$ strength requires estimates of

\footnotetext{
${ }^{7}$ Note that while the expected asymmetry in the location of the stripped material would undoubtedly alter the shape of the resulting line profile, Mattila et al. (2005) conclude that it should not affect the total strength of the line, and so the assumption of spherical symmetry is deemed to be acceptable for the purposes of their study (and, hence, ours).
} 
the distance and extinction of the SNe. For SN 2005am, we adopt the NED Hubble flow $\left(H_{0}=73 \mathrm{~km} \mathrm{~s}^{-1} \mathrm{Mpc}^{-1}\right)$ distance of $36.8 \pm 2.6 \mathrm{Mpc}$ based on the $3 \mathrm{k}$ CMB rest frame as determined by Fixsen et al. (1996), and the total extinction of $A_{V}=0.27 \pm 0.08 \mathrm{mag}$ determined by Li et al. (2006). For SN 2005cf, we use the distance and extinction values adopted by Pastorello et al. (2007) of $D=31.77 \pm 4.8 \mathrm{Mpc}$ and $A_{V}=0.32 \pm 0.03 \mathrm{mag}$. For $0.05 M_{\odot}$ of solar-abundance material 380 days after explosion, we derive expected $\mathrm{H} \alpha$ line fluxes at the profile's peak of $1.72 \times 10^{-18}$ and $2.23 \times 10^{-18} \mathrm{erg} \mathrm{s}^{-1} \AA^{-1} \mathrm{~cm}^{-2}$ for SN $2005 \mathrm{am}$ and SN 2005cf, respectively.

To derive the equivalent width that such features would have had in our observed spectra, we approximated the $\mathrm{H} \alpha$ emission as a Gaussian with a $1 \sigma$ width of $11 \AA$, and placed the resulting profile on top of the estimated "continuum" level at $\lambda=6563 \AA$ (see Fig. 1). We then measured the equivalent width of these theoretical profiles within the region $\pm 11 \AA$ from $\lambda_{0}=6563 \AA$ [the same spectral range used to derive $W_{\lambda}(3 \sigma)$ earlier] in each spectrum. The calculated values for $W_{\lambda}\left(0.05 M_{\odot}\right)$ are given in Table 2, Since the $\mathrm{H} \alpha$ emission is clearly a time-dependent phenomenon and the Mattila et al. (2005) model is specifically for day 380, our estimate of the strength of the line in the day 298 and day 267 spectra of SN 2005am and SN 2005cf, respectively, are quite crude. Fortunately, since the $\mathrm{H} \alpha$ emission is powered primarily by gamma-ray deposition (Mattila et al. 2005), and the optical depth to gamma-rays in the central, hydrogen rich region should be higher at earlier times, we would expect the $\mathrm{H} \alpha$ line to be even stronger at earlier times than it is on day 380 (assuming, of course, that the outer, iron-rich ejecta are transparent at earlier times, and allow us to see it; see $\S[3)$. Thus, our calculated value of $W_{\lambda}\left(0.05 M_{\odot}\right)$ for epochs earlier than 380 days should serve as a conservative lower limit for these spectra.

Finally, we assume a linear scaling between the amount of stripped material and the expected equivalent width of the $\mathrm{H} \alpha$ emission line (an approximation justified through examination of Figure 6 of Mattila et al. 2005, where theoretical profiles are shown for 0.01 and $0.05 M_{\odot}$ ) to arrive at our final estimate for the upper limit on the amount of solar abundance material that could have remained undetected at each spectral epoch. We calculated this limit according to:

$$
\mathrm{M}\left(M_{\odot}\right) \leq \frac{W_{\lambda}(3 \sigma)}{W_{\lambda}\left(0.05 M_{\odot}\right)} \times 0.05
$$

Final results are listed in Table 2, and can be summarized succinctly: We restrict the amount of solar-abundance material that could have evaded detected to be $<0.01 M_{\odot}$ at all spectral epochs except day 384 of SN 2005cf, for which the formal upper limit is $0.02 M_{\odot}$. 


\section{Discussion}

When coupled with the modeling results of Mattila et al. (2005) and the mass-stripping estimates of Marietta et al. (2000) and Meng et al. (2007), our restrictions on $\mathrm{H} \alpha$ emission place strong constraints on the progenitor systems that could have given rise to SN 2005am and SN 2005cf. In short, they rule out all hydrogen-donating companions close enough to the WD to have been experiencing RLOF at the time of explosion (i.e., $a / R<3$; see $\S$ 1). Under the favored single-degenerate scenario, this leaves only widely separated systems in which the companion (i.e., a red giant) donates matter through a strong stellar wind, as a viable option. For the symbiotic case, if we assume that the same scaling between the amount of stripped material and the orbital separation holds for red giants as was found by Marietta et al. (2000) for main-sequence secondaries (see $\S$ 1; note that red giant companions were not analyzed by Meng et al. 2007), then we conclude that the mass-donating red giant must be $\gtrsim 10$ A.U. from the WD to be consistent with our $\mathrm{H} \alpha$ nondetections. For a $1.4 M_{\odot}$ primary and a $1.0 M_{\odot}$ secondary, this corresponds to an orbital period of $\gtrsim 20 \mathrm{yr}$.

Our main conclusion — that the most likely progenitors of SN 2005am and SN 2005cf were widely separated symbiotics — stands in evident contrast to the work of Panagia et al. (2006) on the search for, and subsequent nondetection of, radio emission from SNe Ia. In their study, Panagia et al. (2006) set upper limits on mass-loss rates of $\sim 10^{-7} M_{\odot} \mathrm{yr}^{-1}$ for the progenitor systems. Since this is of the same order of magnitude as the observed massloss rates from symbiotics (e.g., Jung \& Lee 2004; Crowley 2006), Panagia et al. (2006) specifically rule out symbiotics as potential progenitors of SNe Ia. However, the inferences drawn by Panagia et al. (2006) rely on the assumption that SN Ia radio light curves behave identically to those of SNe Ib/c. Such an assumption is necessary because the correlation between wind density and radio luminosity for SNe Ia cannot be calculated from theory at this point. In the absence of theoretical or observational support for such an assumption the very low mass-loss rates claimed by Panagia et al. (2006) cannot yet be considered definitive (Hughes et al. 2007). The radio non-detections may therefore still permit the symbiotic scenario. Along these same lines, the investigation of X-ray non-detections of

SNe Ia by Hughes et al. (2007) provides mass-loss limits of only $\sim 10^{-5} M_{\odot} \mathrm{yr}^{-1}$, which are not stringent enough to rule out symbiotics. While a low-density circumstellar environment is clearly favored by both the radio and X-ray non-detections of SNe Ia, it seems premature to rule out progenitor classes on this basis at the present time.

There are additional caveats to our conclusion that must be given. Of primary significance is the fact that its robustness depends critically on the validity of the theoretical modeling of just a few studies. Taken together, these studies predict that a substantial amount of stripped hydrogen should exist at low velocities (Marietta et al. 2000; Meng et al. 2007) and emit a strong $\mathrm{H} \alpha$ feature at late times (Mattila et al. 2005). There are, however, alternative explanations for the observed lack of hydrogen that must be thoroughly investigated 
to build confidence in the inferences that can be drawn from our study. We now critically evaluate four such alternatives, and point out areas ripe for additional theoretical study.

(1) The hydrogen is "hidden" behind an opaque screen of faster-moving ejecta. Posed simply: Are the fast-moving, iron-rich ejecta truly optically thin at late times? Since permitted Fe II lines dominate the underlying spectrum of SNe Ia near $6563 \AA$, significant opacity in these lines could provide an "iron curtain" that shields the hydrogen emission from our view. Indeed, Kozma et al. (2005) state that several Fe II lines do remain optically thick in their models even at late times. On the other hand, the simulations of Mattila et al. (2005), which predict the strong $\mathrm{H} \alpha$ feature, are explicitly based on the models of Kozma et al. (2005), and so it seems reasonable to infer that the specific Fe II lines near $\mathrm{H} \alpha$ do not provide significant opacity in the Kozma et al. (2005) models. Independent confirmation of the transparency of the outer ejecta of SNe Ia at late times is needed.

(2) There is far less hydrogen entrained in the ejecta than current models predict. By predicting up to a factor of four reduction in the amount of stripped mass compared with the models of Marietta et al. (2000), Meng et al. (2007) demonstrate the potentially large effect that binary evolution can have on the amount of stripped material. Might other aspects of binary evolution be at work to further reduce the stripped mass? A possibly important mechanism not considered by Meng et al. (2007) is the outcome that a strong "accretion wind", blown from the WD during the mass-accretion phase, could have on the secondary's envelope. Accretion winds were originally conceived by Hachisu et al. (1996) as a means to stabilize the mass-transfer process and allow the system to avoid a common envelope phase. Further investigation by Hachisu et al. (1999) and Hachisu \& Kato (2003) revealed that the winds should also heat and ablate a significant amount of material from a close secondary's envelope. From these considerations, Hachisu et al. (1999) estimate that the wind could strip mass from such a companion at rates as high as $\sim 10^{-6} M_{\odot} \mathrm{yr}^{-1}$ for a period of up to $\sim 10^{6} \mathrm{yr}$ prior to the explosion. If such a process actually precedes an SN Ia explosion, it would clearly leave the secondary's envelope in a vastly different state from the models considered to date. In fact, such secondaries could potentially lose their entire hydrogen envelope and evolve to become helium-donators, which would naturally account for a lack of hydrogen in late-time spectra (Branch et al. 1995).

There are both theoretical and observational objections to strong WD winds playing a large roll in stripping mass from the secondary star in SN Ia progenitor systems, however. On the theoretical front, Livio (2001) questions the high efficiency of the mass stripping proposed by Hachisu et al. (1999), and also points out that at high accretion rates, the bulk of the WD's wind may be strongly collimated in a direction perpendicular to the accretion disk rather than in the direction of the companion star. Such a scenario would result in little stripping. Observational doubt on accretion winds playing a major role in pre-SN Ia evolution is cast by Badenes et al. (2007), who find that such an optically thick outflow from a WD's surface should excavate a large, low-density cavity around the system. 
Such a large evacuated region is incompatible with the known observational properties of SN Ia remnants in the Galaxy, the Large Magellanic Cloud, and M31. It thus appears that, while such accretion winds may occur in nature (e.g., Hachisu \& Kato 2003), they do not evidently precede a majority of SN Ia explosions. Further consideration of this and other potential mass-stripping mechanisms is certainly warranted.

(3) The hydrogen gas is insufficiently powered to produce $H \alpha$ emission. Mattila et al. (2005) conclude that the hydrogen-rich, central region of the young supernova remnant presents high optical depth to gamma-ray photons that originate from the radioactive decay of isotopes synthesized in the explosion. The gamma-ray trapping is then responsible for powering the $\mathrm{H} \alpha$ line emission. This mechanism is quite different from the situation in the outer, lower density regions of the ejecta, whose optical emission is predominantly powered by local positron deposition (e.g., Stritzinger \& Sollerman 2007). Since the production of a strong $\mathrm{H} \alpha$ line from even small amounts of solar-abundance material relies fundamentally on the inner ejecta's ability to trap gamma rays, the high opacity of the material, and the existence of sufficient numbers of gamma-rays to power the emission, should be confirmed by independent modeling.

(4) The single-degenerate scenario is not responsible for these SNe Ia. A doubledegenerate progenitor system, in which two WDs in a binary system coalesce due to the

emission of gravitational radiation and ultimately explode as an SN Ia (Webbink 1984; Iben \& Tutukov 1984), would naturally account for the absence of hydrogen in the spectra. Indeed, detection of any hydrogen would deal a death blow to the double-degenerate scenario. Difficulties with the double-degenerate scenario are well known (see Livio 2001 for a thorough review), however, and include both observational (e.g., of the $\sim 120$ doubledegenerate systems known, none have a total mass in excess of the Chandrasekhar limit; Napiwotzki et al. 2004) and theoretical (e.g., the merger process seems more likely to lead to collapse to a neutron star than to thermonuclear explosion as an SN Ia; Saio \& Nomoto 1998, and references therein) objections. Nonetheless, theoretical loopholes remain (e.g., Piersanti et al. 2003; Yoon et al. 2007), and the observational sample of nearby doubledegenerate systems is not complete (Nelemans et al. 2005). Since the double-degenerate channel is a potential "silver-bullet" that can explain both the lack of hydrogen in SNe Ia as well as the occurrence of SNe Ia in both old and young star-forming systems (e.g., Branch et al. 1995), it requires continued theoretical and observational attention.

\section{Conclusions}

We obtained five deep, moderate-resolution, nebular-phase spectra of two SNe Ia (SN 2005am and SN 2005cf) in order to search for narrow $\mathrm{H} \alpha$ emission that would betray the existence of material stripped from the envelope of a mass-donating stellar companion to the exploding WD. No such emission is detected in either object at any epoch. From the 
models of Mattila et al. (2005), we establish upper limits of $0.01 M_{\odot}$ of solar abundance material in the inner ejecta of both objects, which are the tightest constraints yet established by such studies. Our non-detections of $\mathrm{H} \alpha$, coupled with the mass-stripping results of Marietta et al. (2000) and Meng et al. (2007), rule out all hydrogen-donating companions close enough to the WD to have been experiencing RLOF at the time of explosion for these events. Additional theoretical work is needed in several areas to buttress this conclusion, including most critically verification of the transparency of the outer, more rapidly moving ejecta that could potentially block $\mathrm{H} \alpha$ photons from escaping from the inner region. Bearing this caveat in mind, we propose that symbiotics are, at this time, the most likely progenitor class that remains consistent with these data.

Definitive proof of the identity of the progenitor system(s) that gives rise to SNe Ia remains elusive, and it must be admitted that our conclusion, which is based on the lack of a detection, is not as satisfying as one based on a detection. Should future modeling efforts prove unable to "hide the hydrogen" for even widely separated binaries, then the continued viability of the single-degenerate, hydrogen-donating progenitor will require that $\mathrm{H} \alpha$, no matter how weak, must ultimately be detected.

I owe a debt of gratitude to Tom Matheson for valuable assistance with the acquisition and reduction of the data obtained with the Gemini telescopes. I acknowledge support from an NSF Astronomy and Astrophysics Postdoctoral Fellowship (award AST-040147), under which part of this research was carried out. This research has made use of the NASA/IPAC Extragalactic Database (NED), which is operated by the Jet Propulsion Laboratory, California Institute of Technology, under contract with NASA. Finally, I wish to recognize and acknowledge the very significant cultural role and reverence that the summit of Mauna Kea has always had within the indigenous Hawaiian community. We are most fortunate to have the opportunity to conduct observations from this mountain.

\section{REFERENCES}

Badenes, C., Hughes, J. P., Bravo, E., \& Langer, N. 2007, ApJ, 662, 472

Baldwin, J. A., \& Stone, R. P. S. 1984, MNRAS, 206, 241

Benetti, S., et al. 2004, MNRAS, 348, 261

Branch, D., Livio, M., Yungelson, L. R., Boffi, F. R., \& Baron, E. 1995, PASP, 107, 1019

Brown, P. J., et al. 2005, ApJ, 635, 1192

Cappellaro, E., et al. 2001, ApJ, 549, L215

Chugai, N. N. 1986, Soviet Astronomy, 30, 563

Crowley, C. 2006, Ph.D. thesis, Trinity College Dublin

Eggleton, P. P. 1973, MNRAS, 163, 279

-. 1983, ApJ, 268, 368 
Elias-Rosa, N., et al. 2006, MNRAS, 369, 1880

Filippenko, A. V. 1982, PASP, 94, 715

Fixsen, D. J., Cheng, E. S., Gales, J. M., Mather, J. C., Shafer, R. A., \& Wright, E. L. 1996, ApJ, 473, 576

Fryxell, B. A., \& Arnett, W. D. 1981, ApJ, 243, 994

Hachisu, I., \& Kato, M. 2003, ApJ, 590, 445

Hachisu, I., Kato, M., \& Nomoto, K. 1996, ApJ, 470, L97+

-. 1999, ApJ, 522, 487

Hook, I. M., Jørgensen, I., Allington-Smith, J. R., Davies, R. L., Metcalfe, N., Murowinski, R. G., \& Crampton, D. 2004, PASP, 116, 425

Horne, K. 1986, PASP, 98, 609

Hughes, J. P., Chugai, N., Chevalier, R., Lundqvist, P., \& Schlegel, E. 2007, ApJ, in press Iben, Jr., \& \& Tutukov 1984, ApJS, 54, 335

Jung, Y.-C., \& Lee, H.-W. 2004, MNRAS, 355, 221

Kozma, C., Fransson, C., Hillebrandt, W., Travaglio, C., Sollerman, J., Reinecke, M., Röpke, F. K., \& Spyromilio, J. 2005, A\&A, 437, 983

Lair, J. C., Leising, M. D., Milne, P. A., \& Williams, G. G. 2006, AJ, 132, 2024

Leonard, D. C., \& Filippenko, A. V. 2001, PASP, 113, 920

Li, W., Jha, S., Filippenko, A. V., Bloom, J. S., Pooley, D., Foley, R. J., \& Perley, D. A. 2006, PASP, 118, 37

Livio, M. 2001, in Supernovae and Gamma-Ray Bursts: the Greatest Explosions since the Big Bang. Proceedings of the Space Telescope Science Institute Symposium, May 3 - 6, 1999, ed. M. Livio, N. Panagia, \& K. Sahu (Cambridge: Cambridge University Press), 334

Livne, E., Tuchman, Y., \& Wheeler, J. C. 1992, ApJ, 399, 665

Marietta, E., Burrows, A., \& Fryxell, B. 2000, ApJS, 128, 615

Massey, P., \& Gronwall, C. 1990, ApJ, 358, 344

Matheson, T., Filippenko, A. V., Ho, L. C., Barth, A. J., \& Leonard, D. C. 2000, AJ, 120, 1499

Mattila, S., Lundqvist, P., Sollerman, J., Kozma, C., Baron, E., Fransson, C., Leibundgut, B., \& Nomoto, K. 2005, A\&A, 443, 649

Meng, X., Chen, X., \& Han, Z. 2007, PASJ, 706, in press (arXiv:0706.3581v1)

Munari, U., \& Renzini, A. 1992, ApJ, 397, L87

Napiwotzki, R., et al. 2004, in ASP Conf. Ser. 318: Spectroscopically and Spatially Resolving the Components of the Close Binary Stars, ed. R. W. Hilditch, H. Hensberge, \& K. Pavlovski (San Francisco: ASP), 402

Nelemans, G., et al. 2005, A\&A, 440, 1087

Oke, J. B., \& Gunn, J. E. 1983, ApJ, 266, 713

Oke, J. B., et al. 1995, PASP, 107, 375

Panagia, N., Van Dyk, S. D., Weiler, K. W., Sramek, R. A., Stockdale, C. J., \& Murata, K. P. 2006, ApJ, 646, 369 
Pastorello, A., et al. 2007, MNRAS, 376, 1301

Piersanti, L., Gagliardi, S., Iben, I. J., \& Tornambé, A. 2003, ApJ, 583, 885

Press, W. H., Teukolsky, S. A., Vetterling, W. T., \& Flannery, B. P. 1992, Numerical Recipes in C: The Art of Scientific Computing (2d ed.; Cambridge: Cambridge Univ. Press)

Saio, H., \& Nomoto, K. 1998, ApJ, 500, 388

Stanishev, V., et al. 2007, A\&A, 469, 645

Stritzinger, M., \& Sollerman, J. 2007, A\&A, 470, L1

Taam, R. E., \& Fryxell, B. A. 1984, ApJ, 279, 166

Webbink, R. F. 1984, ApJ, 277, 355

Wheeler, J. C., Lecar, M., \& McKee, C. F. 1975, ApJ, 200, 145

Whelan, J., \& Iben, I. J. 1973, ApJ, 186, 1007

Yoon, S.-C., Podsiadlowski, P., \& Rosswog, S. 2007, MNRAS, 380, 933 


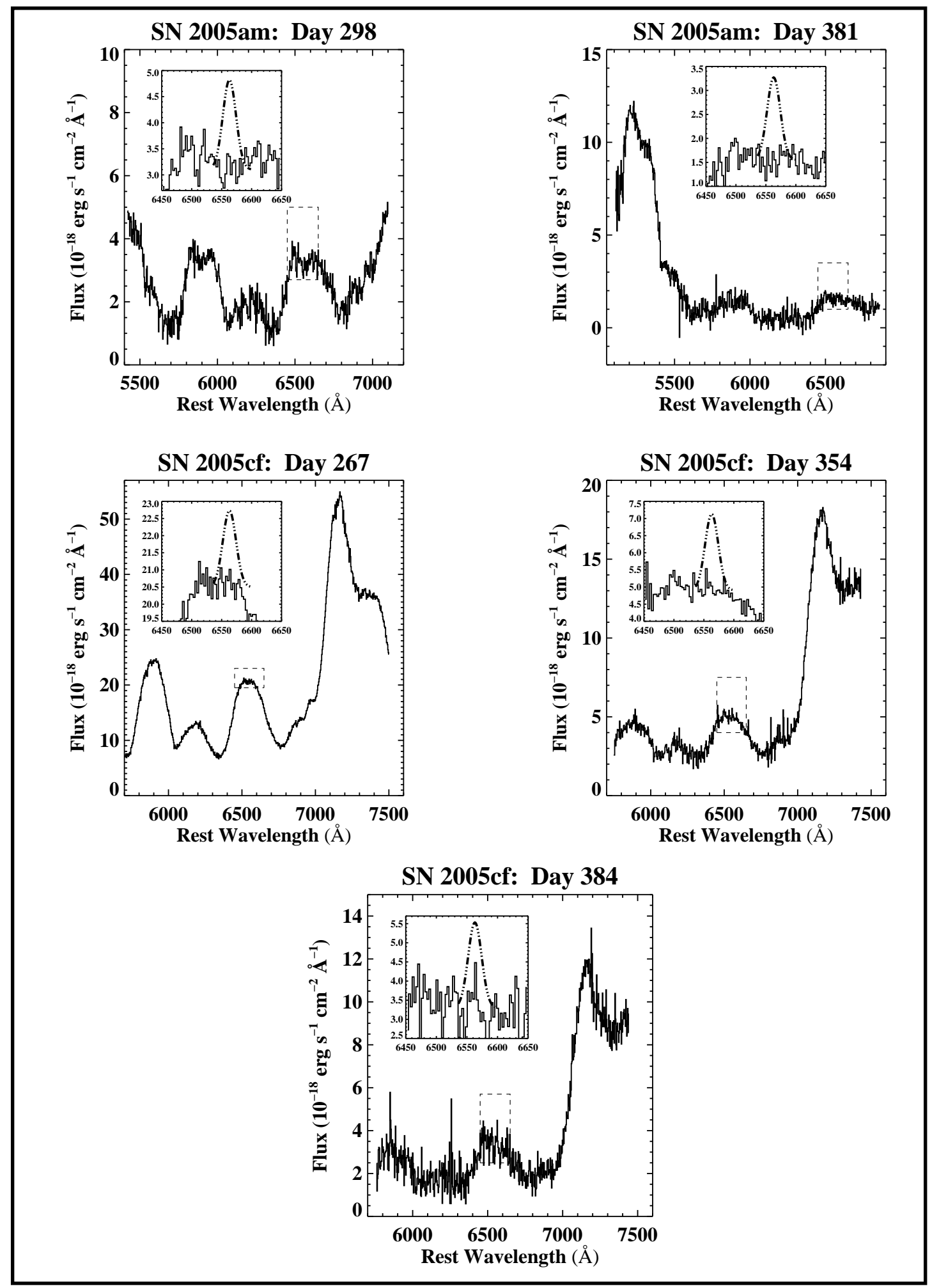

Fig. 1. - Late-time spectra of two SNe Ia, with day since $B$ maximum indicated. The spectra are displayed at $3 \AA \mathrm{bin}^{-1}$, the approximate resolution at $\mathrm{H} \alpha$. The expected strengths of the $\mathrm{H} \alpha$ line resulting from $0.05 M_{\odot}$ of solar-abundance material according to the day 380 models of Mattila et al. (2005) are shown as dot-dashed lines in the insets; note that since the $\mathrm{H} \alpha$ emission is a time-dependent phenomenon, the estimated strength of these lines in the day 298 and day 267 spectra of SN 2005am and SN 2005cf, respectively, are only approximate. 
Table 1. Journal of Spectroscopic Observations

\begin{tabular}{|c|c|c|c|c|c|c|c|c|c|c|c|c|c|}
\hline UT Date & Object & Day $^{\mathrm{a}}$ & $\begin{array}{c}\text { HJD } \\
-2,400,000\end{array}$ & Telescope $^{\mathrm{b}}$ & $\begin{array}{c}\text { Range }^{c} \\
(\AA)\end{array}$ & $\begin{array}{l}\text { Resolution }{ }^{\mathrm{d}} \\
(\AA)\end{array}$ & $\begin{array}{l}\text { P.A. }^{\text {e }} \\
(\mathrm{deg})\end{array}$ & $\begin{array}{l}\text { Par. P.A. }{ }^{\mathrm{f}} \\
\quad(\mathrm{deg})\end{array}$ & Air Mass ${ }^{g}$ & $\begin{array}{c}\text { Flux } \\
\text { Standard }^{\mathrm{h}}\end{array}$ & $\begin{array}{l}\text { Seeing }{ }^{\mathrm{i}} \\
(\operatorname{arcsec})\end{array}$ & $\begin{array}{c}\text { Slit } \\
(\operatorname{arcsec})\end{array}$ & $\begin{array}{c}\text { Exposure } \\
\text { (s) }\end{array}$ \\
\hline 2005 Dec 31.52 & SN 2005am & 298.02 & 53736.02 & $\mathrm{KI}$ & $5860-7160$ & 3.0 & 150 & $149-4$ & $1.24-1.32$ & HD84 & 1.3 & 1.0 & 7,200 \\
\hline 2006 Mar 6.59 & SN $2005 \mathrm{cf}$ & 267.09 & 53801.09 & GN & $5520-7628$ & 3.1 & 55 & $135-22$ & $1.12-1.33$ & $\mathrm{HZ} 44$ & 0.7 & 1.0 & 10,800 \\
\hline 2006 Mar 24.15 & SN 2005am & 380.65 & 53818.65 & GS & $5150-6920$ & 3.2 & 0 & $117-156$ & $1.04-1.34$ & LTT1020 & 0.7 & 1.0 & 10,410 \\
\hline 2006 Jun 1.46 & SN $2005 \mathrm{cf}$ & 353.96 & 53887.96 & KI & $5750-7430$ & 2.4 & 55 & $21-58$ & $1.15-1.70$ & BD26 & 1.1 & 1.0 & 11,100 \\
\hline 2006 Jul 1.39 & SN 2005cf & 383.89 & 53917.89 & KI & $5796-7496$ & 3.4 & 55 & $47-61$ & $1.32-1.93$ & BD26 & 1.0 & 1.5 & 6,600 \\
\hline
\end{tabular}

Note. - All Keck observations were obtained with the 900/5500 [number of lines $\mathrm{mm}^{-1}$ /blaze wavelength $(\AA)$ ] grating + GG495 order blocking filter; all Gemini observations were carried out with the R831_G5322 grating + OG515_GO330 order blocking filter. Gemini observations were conducted under programs GS-2006A-Q-28 (SN 2005am; PI: Leonard) and GN-2006A-Q-27 (SN 2005cf; PI: Leonard).

a Days since estimated dates of maximum $B$ brightness. SN 2005am: 2005 March 8.5 \pm 1 , HJD 2, 453, $438 \pm 1$ (Brown et al. 2005). SN 2005cf: 2005 June 12.5 \pm 0.3 , HJD 2, 453, 534.0 0.3 (Pastorello et al. 2007).

${ }^{\mathrm{b}} \mathrm{KI}=$ Keck I $10 \mathrm{~m} /$ Low-Resolution Imaging Spectrometer + polarimeter (LRISp; Oke et al. 1995); GN(S) = Gemini North (South) 8 m/Gemini Multi-Object Spectrograph (GMOS; Hook et al. 2004).

${ }^{\mathrm{c}}$ Wavelength range of the calibrated flux spectrum.

${ }^{\mathrm{d}}$ Approximate spectral resolution derived from night-sky lines near the nominal location of $\mathrm{H} \alpha$ in the observed spectrum.

${ }^{\text {e }}$ Position angle of the spectrograph slit.

${ }^{\mathrm{f}}$ Parallactic angle (Filippenko 1982) range calculated at the midpoint of each separate observation for each set of observations.

${ }^{\mathrm{g}}$ Airmass range calculated at the midpoint of each separate observation for each set of observations.

${ }^{\mathrm{h}} \mathrm{HD} 84=\mathrm{HD}$ 84937, BD26 $=\mathrm{BD}+26^{\circ} 2606$ (Oke \& Gunn 1983); HZ44 = Hz 44 (Massev \& Gronwall 1990); LTT1020 = LTT 1020 (Baldwin \& Stone 1984). Standard stars for observations made at the Keck Observatory were observed on the same night as the supernova observations; for the Gemini Observatory observations, the standard stars were observed on 2006 February 15 (HZ44, for the day 267 observation of SN 2005cf) and 2005 September 5 (LTT 1020, for the day 381 observations of SN 2005am). The observations of LTT 1020 were made as part of program GS-2005B-C-4 (PI: Dan Christlein).

${ }^{i}$ Average value of the full width at half maximum of the spatial profile for each set of observations, rounded to the nearest $0^{\prime \prime} 1$.

${ }^{\mathrm{j}}$ Combined exposure duration of all observations, in seconds. Observations at the Keck Observatory consisted of four separate, successive exposures, while observations at the Gemini Observatories consisted of six separate, successive exposures. 
Table 2. Measured Values

\begin{tabular}{lccccccc}
\hline \hline \multicolumn{1}{c}{ Object } & Day & Factor $^{\mathrm{a}}$ & $B^{\mathrm{b}}$ & $\Delta I^{\mathrm{c}}$ & $\begin{array}{c}W_{\lambda}(3 \sigma)^{\mathrm{d}} \\
(\AA)\end{array}$ & $\begin{array}{c}W_{\lambda}\left(0.05 M_{\odot}\right)^{\mathrm{e}} \\
(\AA)\end{array}$ & $\mathrm{M}\left(M_{\odot}\right)^{\mathrm{f}}$ \\
\hline SN 2005am & 298.02 & 1.16 & 1.00 & 0.090 & 2.19 & 10.76 & 0.01 \\
SN 2005cf & 267.09 & 1.45 & 0.97 & 0.015 & 0.38 & 2.11 & 0.01 \\
SN 2005am & 380.65 & 0.45 & 0.94 & 0.144 & 3.73 & 21.51 & 0.01 \\
SN 2005cf & 353.96 & 1.22 & 1.25 & 0.055 & 1.07 & 8.82 & 0.01 \\
SN 2005cf & 383.89 & 1.02 & 0.88 & 0.164 & 4.51 & 13.10 & 0.02 \\
\hline
\end{tabular}

Note. - All measurements made on rest-frame spectra rebinned to $3 \AA \mathrm{bin}^{-1}$.

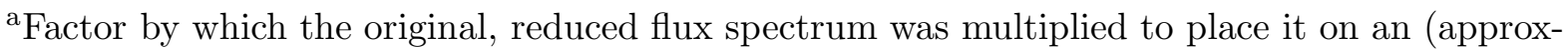
imate) absolute flux scale; see text for details.

bNumber of $3 \AA$ bins per resolution element.

${ }^{\mathrm{c}} 1 \sigma$ root-mean-square fluctuation of the flux around a normalized continuum.

${ }^{\mathrm{d}}$ Equivalent width of the strongest potential feature near $\lambda_{0}=6563 \AA$ that could have remained undetected in the spectrum, derived using Equation 1 .

${ }^{\text {e}}$ Expected equivalent width of an $\mathrm{H} \alpha$ emission line resulting from $0.05 M_{\odot}$ of solar abundance material according to the models of Mattila et al. (2005); see text for details.

${ }^{\mathrm{f}}$ Derived upper limit (rounded up to the nearest $0.01 M_{\odot}$ ) on the amount of solar abundance material that could have remained undetected at each spectral epoch, derived according to Equation 2 . 